\title{
QUANTUM STATISTICS \\ OF METASTABLE LIQUID HELIUM
}

\author{
FRÉDÉRIC CAUPIN AND SÉBASTIEN BALIBAR \\ Laboratoire de Physique Statistique \\ de l'Ecole Normale Supérieure \\ associé aux Universités Paris 6 et Paris 7 et au CNRS \\ 24 rue Lhomond 75231 Paris Cedex 05, France
}

\begin{abstract}
Experimental studies of homogeneous cavitation give information on the limit of stability of liquids, the "spinodal limit". The slope of the spinodal line in the $(P-T)$ plane has been related to the sign of the isobaric expansion coefficient of the liquid. Using quantum statistics, we give theoretical arguments to explain the existence of a minimum in the spinodal line of liquid helium 3, for which experimental evidence has been reported. The calculations involve the shape of the dispersion curve of collective excitations. We present a similar analysis in the case of superfluid helium 4; we also consider the overpressurized region, and the location of the superfluid transition line in the metastable regions.
\end{abstract}

\section{Introduction}

We have investigated homogeneous cavitation in liquid helium by using a high amplitude ultrasonic wave generated by a hemispherical transducer [1, $2,3]$. This brings the liquid in a metastable state, because at pressures below the saturated vapour pressure (svp), an energy barrier needs to be overcome for the gas phase to nucleate. This barrier vanishes at a negative pressure called the spinodal pressure $P_{\mathrm{s}}$, where the compressibility of the liquid diverges (the sound velocity vanishes). Cavitation becomes likely when the wave produces a sufficiently large negative pressure swing, corresponding to the cavitation pressure $P_{\text {cav }}$.

In liquid helium 4, we had previously found a crossover from a high temperature regime where cavitation is a thermally activated process, to a low temperature regime where it occurs by quantum tunneling $[1,2$, $3]$. In liquid helium 3, where this crossover was predicted to take place at $120 \mathrm{mK}[4,5]$, measurements performed down to $35 \mathrm{mK}$ have shown a different behaviour $[2,6]$. To explain this, we have proposed to consider 
the Fermi degeneracy of helium 3 [7]. Here we will focus on the thermally activated regime.

In liquid helium 3, the experimental temperature dependence of $P_{\text {cav }}$ supports the existence of a minimum at $0.4 \mathrm{~K}$ in the liquid-gas spinodal line $P_{\mathrm{S}}(T)$. R. J. Speedy previously proposed this peculiar behaviour for the spinodal of water and related it to a change in sign of the expansion coefficient $\alpha$, i. e. a line of density maxima (LDM) [8]. In Sec. 2 we show that this feature is consistent with extrapolations of sound velocity measurements and calculate $\alpha$ near the spinodal line.

We consider stretched liquid helium 4 in Sec. 3. After giving a short review of previous work, we use quantum statistics to derive $\alpha$ and the shape of the spinodal, and to describe the superfluid transition line at negative pressure.

In Sec. 4 we finally consider overpressurized helium 4 and we predict some properties of the phase diagram in this other metastable region.

\section{Liquid helium 3 at negative pressure}

In this section we summarize a recent article written with H.J. Maris [9] before giving more details on the calculations involving quantum statistics.

\subsection{EXPERIMENTAL EVIDENCE FOR A MINIMUM IN THE SPINODAL}

We have performed experiments in a volume $V$ and during a time $\tau$ and measured the cavitation probability $\Sigma[2]$. At a pressure $P$ and a temperature $T$, the energy barrier that must be overcome for liquid-gas separation to occur is $E_{\mathrm{b}}(P, T)$, and we have:

$$
\Sigma(P, T)=1-\exp \left[-\Gamma_{0} V \tau \exp \left(-\frac{E_{\mathrm{b}}(P, T)}{k_{\mathrm{B}} T}\right)\right]
$$

where $\Gamma_{0}$ has the dimensions of frequency times an inverse volume. It is thus natural to estimate $\Gamma_{0}$ as an attempt frequency $\nu$ at which the fluctuations try to overcome the nucleation barrier multiplied by the density of the critical nuclei which can be taken to be spheres of radius $R_{\mathrm{c}}[10,11,12]$. Typically, $R_{\mathrm{c}}$ is around $1 \mathrm{~nm}$ and the attempt frequency varies from $k_{\mathrm{B}} T / h$ to $E_{\mathrm{b}} / h$; all the different estimates thus lie between $5 \times 10^{36} \mathrm{~T}$ and $1.5 \times$ $10^{38} \mathrm{~T} \mathrm{~m}^{-3} \mathrm{~s}^{-1} \mathrm{~K}^{-1}$. Pettersen et al. [12] have calculated $V$ and $\tau$ for the experimental method which uses an acoustic wave to produce a negative pressure swing in the liquid. For ${ }^{3} \mathrm{He}$ and for a $1 \mathrm{MHz}$ acoustic wave as in Ref. [2], this gives $V \tau=1.2 \times 10^{-22} \mathrm{~m}^{3} \mathrm{~s}$ [13]. The theoretical estimates of the factor $\Gamma_{0} V \tau$ thus vary from $6 \times 10^{14} T$ to $1.8 \times 10^{16} T \mathrm{~K}^{-1}$. Although this range extends over two orders of magnitude, it does not significantly 


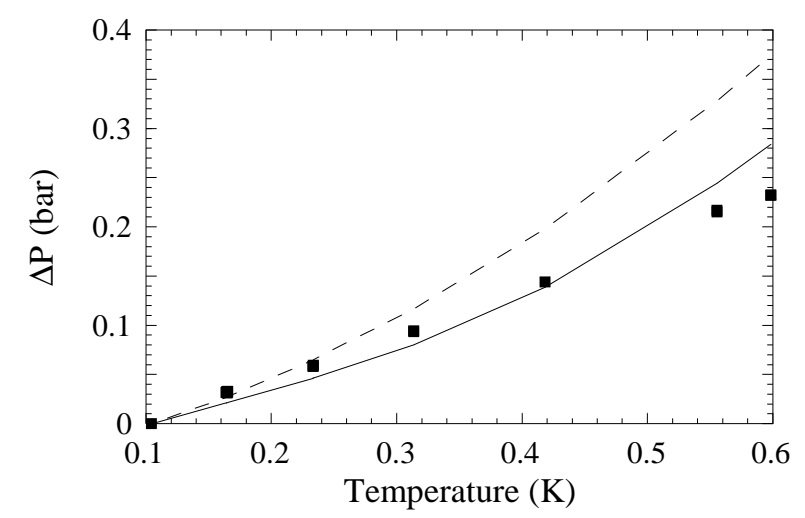

Figure 1. Temperature variation $\Delta P=P_{\text {cav }}(T)-P_{\text {cav }}(0.1 \mathrm{~K})$ of the cavitation pressure. Experimental $\Delta P$ is given by solid squares. Other lines are calculated $\Delta P$ according to Guilleumas et al. [11] (dashed line) and this work (solid line).

affect the value of the energy barrier: for $\Sigma=0.5$, all estimates give $E_{\mathrm{b}}=$ $(34 \pm 3) k_{\mathrm{B}} T$.

Maris [4] has calculated $E_{\mathrm{b}}(P)$ at low temperature by a density functional method; close to the spinodal pressure $P_{\mathrm{s}}$, his results are well represented by a power law:

$$
\frac{E_{\mathrm{b}}}{k_{\mathrm{B}}}=\beta\left(P-P_{\mathrm{s}}\right)^{\delta}
$$

with $\beta=47.13 \mathrm{~K} \mathrm{bar}^{-3 / 4}$ and $\delta=3 / 4$. However, to calculate the cavitation pressure up to $0.6 \mathrm{~K}$, we need to know the temperature dependence of $E_{\mathrm{b}}$. The strongest source of this dependence is that the spinodal pressure varies with temperature; therefore we write $E_{\mathrm{b}}(P, T)=E_{\mathrm{b}}\left(P-P_{\mathrm{S}}(T)\right)$ and assume that Eq. 2 remains valid at higher temperature with parameters $\beta$ and $\delta$ held constant. The temperature dependence of the cavitation pressure follows from Eq. 1:

$$
P_{\text {cav }}(T)=P_{\mathrm{s}}(T)+\left[\frac{T}{\beta} \ln \left(\frac{\Gamma_{0} V \tau}{\ln 2}\right)\right]^{1 / \delta}
$$

To compare the experimental and theoretical temperature variations of $P_{\text {cav }}$, we have plotted the quantity $\Delta P=P_{\text {cav }}(T)-P_{\text {cav }}(0.1 \mathrm{~K})$ in Fig. 1 , using two different spinodal lines $P_{\mathrm{S}}(T)$ and the lowest estimate of the prefactor, namely $\Gamma_{0} V \tau=6 \times 10^{14} \mathrm{~T} \mathrm{~K}^{-1}$. The dashed line was deduced from Eq. 3 using the spinodal line calculated by Guilleumas et al. [11], which is displayed on Fig. 2. It does not agree with the experimental results; to do so, it would require $\Gamma_{0} V \tau$ to be at least 3 orders of magnitude smaller than expected. We do not see any reasons to support this hypothesis. and we think that the discrepancy comes from the shape of the spinodal limit. 


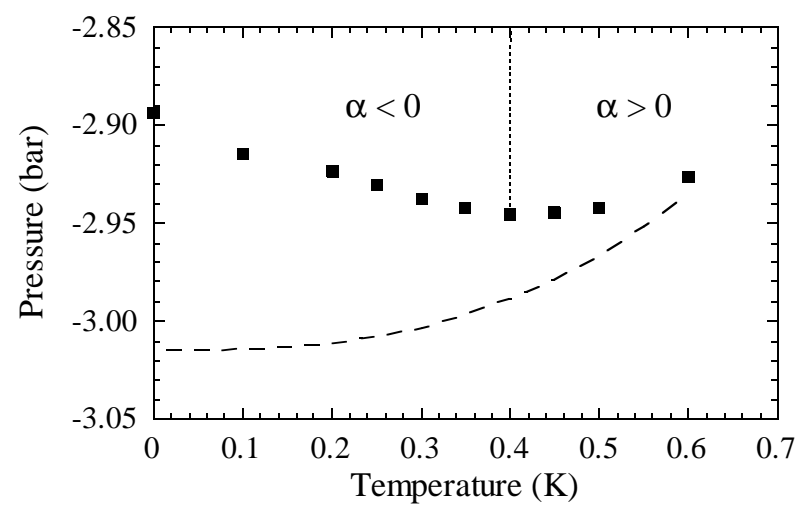

Figure 2. Comparison between two theoretical estimates of the spinodal line: Guilleumas et al. [11] (dashed line) and this work (solid squares). The spinodal found in this work exhibits a minimum at $0.4 \mathrm{~K}$. The dotted line is a linear extrapolation of the LDM as measured by Boghosian et al. [14] between 0 and 11 bar (see Fig. 3).

Before proceeding further, we need to recall how the spinodal pressure $P_{\mathrm{s}}$ can be obtained: Maris' method $[15,4]$ consists in extrapolating measurements of the sound velocity $c$ at positive pressure with a law of the form $c=\left[b\left(P-P_{\mathrm{s}}\right)\right]^{1 / 3}$. Maris used for $c$ the measurements of Abraham et al. at low temperature [16]. We used the same method with a set of data from Roach et al. [17] between 0.01 and $0.6 \mathrm{~K}$ [18] . The spinodal line we obtained is shown in Fig. 2: the spinodal pressure reaches a minimum of -2.9 bar around $T=0.4 \mathrm{~K}$. The new shape of the spinodal curve we propose is sufficient to remove the discrepancy stated above: using again Eq. 3 with the value $\Gamma_{0} V \tau=6 \times 10^{14} \mathrm{TK}^{-1}$, we find a cavitation line which has a temperature dependence consistent with the experimental results (solid line in Fig. 1).

Let us now turn to the physical origin of such a minimum in the spinodal. A similar behavior was first proposed by Speedy in the case of water [8]. A review of related topics also describing alternative theories can be found in Ref. [19]. Following a thermodynamical analysis first developed in the case of helium [20], Speedy shows that close to the spinodal the sign of the isobaric thermal expansion coefficient $\alpha$ of the liquid is the same as the sign of $\mathrm{d} P_{\mathrm{S}} / \mathrm{d} T$. Therefore, if the locus of points such that $\alpha(P, T)=0$ intersects the spinodal, this results in an extremum in the curve $P_{\mathrm{S}}(T)$. Water and ${ }^{3} \mathrm{He}$ have in common that both liquids exhibit a LDM: in some temperature range, they expand upon cooling. Therefore they may exhibit such a minimum in the spinodal. To obtain the LDM in ${ }^{3} \mathrm{He}$, we used measurements by Boghosian et al. [14], which extend to higher temperatures and agree well with Roach values in the region where both sets overlap; the result is shown in Fig. 3. A simple linear extrapolation of the LDM for 


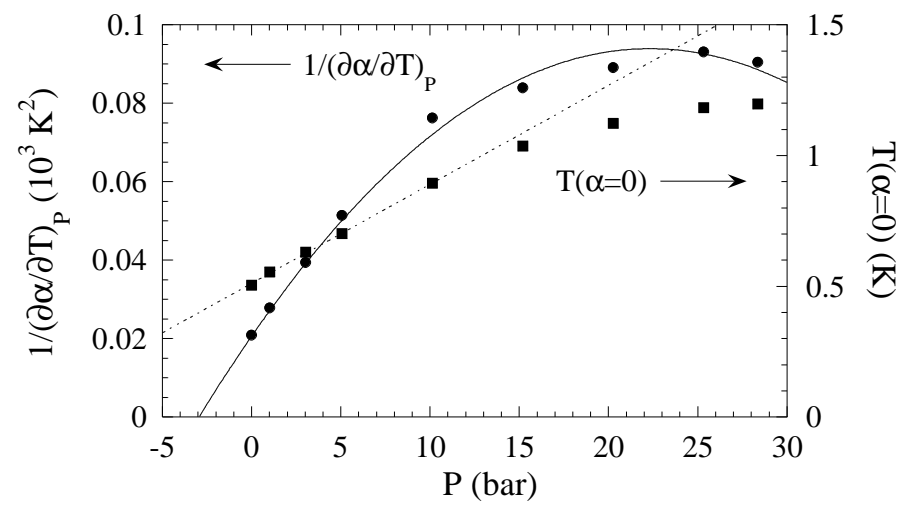

Figure 3. Temperature of density maximum (solid squares) and inverse of $(\partial \alpha / \partial T)_{\mathrm{P}}$ around $\alpha=0$ (solid circles) as functions of pressure, derived from measurements by Boghosian et al. [14]. The dotted line shows the extrapolation of the LDM used in Fig. 2. The solid line is a parabolic fit to $1 /(\partial \alpha / \partial T)_{\mathrm{P}}$ forced to vanish at the pressure of the minimum in the spinodal.

pressures below 11 bar extends down to the minimum in the spinodal as shown in Fig. 2. In his original paper [8], Speedy shows that the expansion coefficient at the spinodal undergoes a jump from $-\infty$ to $+\infty$ at the temperature at which the LDM meets the spinodal. To find some evidence to support this prediction, we follow Speedy's analysis for water and derive the slope $(\partial \alpha / \partial T)_{\mathrm{P}}$ around $\alpha=0$ for each isobar in the measurements by Boghosian et al. This slope should diverge when the pressure reaches the spinodal. Fig. 3 shows that the experimental values are consistent with this prediction.

\subsection{INTERPRETATION}

We now give theoretical arguments to explain why $\alpha$ should be negative in helium 3 at low temperature and near the spinodal. The negative sign of $\alpha$ in ${ }^{3} \mathrm{He}$ was first observed experimentally in 1958 by Lee et al. [21]. The same year, Brueckner and Atkins [22] pointed out how this behaviour was related to the variation of the effective mass $m^{*}$ with the density $\rho$. Indeed, using a Maxwell relation, we can write:

$$
\alpha=-\frac{1}{V}\left(\frac{\partial S}{\partial P}\right)_{T}=-\frac{\rho^{2}}{M} \chi_{T}\left(\frac{\partial S}{\partial \rho}\right)_{T}
$$

At low temperature, the heat capacity of a Fermi gas is:

$$
C_{\mathrm{F}}=\left(\frac{k_{\mathrm{B}}}{\hbar}\right)^{2}\left(\frac{\pi m}{3 \rho}\right)^{2 / 3} T
$$


Because of interactions, helium 3 must be described by the Landau theory of a Fermi liquid [23]. This leads to a corrective factor in the heat capacity $C_{\mathrm{V}}=\left(m^{*} / m\right) C_{\mathrm{F}}$, so that we have $S=C_{\mathrm{V}}=\left(m^{*} / m\right) C_{\mathrm{F}}$. Using Greywall's measurements of the effective mass [24] and extrapolating them at negative pressure as we did before [25], we find that the corresponding $\alpha_{\mathrm{F}}$ given by Eq. 4 remains negative down to the spinodal; when $P \rightarrow P_{\mathrm{s}}$, it diverges as $-T \chi_{T}$.

We now consider the corrections to the linear regime of the heat capacity and their evolution close to the spinodal. We see two sources of corrections. At positive pressure, the main one comes from the coupling of the Landau quasiparticles to the incoherent spin fluctuations and varies as $T^{3} \ln T$. This effect has been studied by Greywall [26], who has shown that its amplitude decreases when pressure decreases; we assume that this is the case until the spinodal is reached, so that $\alpha_{\text {spin }}$ remains negligible compared to $\alpha_{\mathrm{F}}$. The second correction $\alpha_{\mathrm{ph}}$ comes from the contribution of phonons to the heat capacity. The phonons obey Bose statistics and can be described with the grand canonical formalism, using the grand potential

$$
J=\frac{M k_{\mathrm{B}} T}{2 \pi^{2} \rho} \int_{0}^{+\infty} k^{2} \ln \left[1-\exp \left(-\frac{\varepsilon(k)}{k_{\mathrm{B}} T}\right)\right] \mathrm{d} k
$$

where $\varepsilon(k)$ is the energy of a phonon of momentum $k$. The usual linear dispersion relation writes as:

$$
\varepsilon(k)=\hbar c k
$$

where $c$ is the sound velocity. The entropy is deduced by

$$
S=-\left(\frac{\partial J}{\partial T}\right)_{P}
$$

Using Eqs. 4, 6, 8, and 7, one obtains

$$
\alpha_{\mathrm{ph}}=\frac{2 \pi^{2} k_{\mathrm{B}}{ }^{4}}{15 \hbar^{3}}\left(\frac{T}{c}\right)^{3} \chi_{T}\left[\frac{1}{3}+\frac{\rho}{c}\left(\frac{\partial c}{\partial \rho}\right)_{T}\right]
$$

This term is therefore usually neglected compared to $\alpha_{\mathrm{F}}$ (linear in $T$ ), but it could become large near the spinodal where the isothermal sound velocity vanishes. However, this is relevant only for the long wavelength phonons: as stated by Lifshitz and Kagan [27], the first correction to the linear dispersion gives for the energy $\varepsilon(k)$ :

$$
\varepsilon(k)=\hbar k \sqrt{c^{2}+2 \rho \lambda k^{2}}
$$



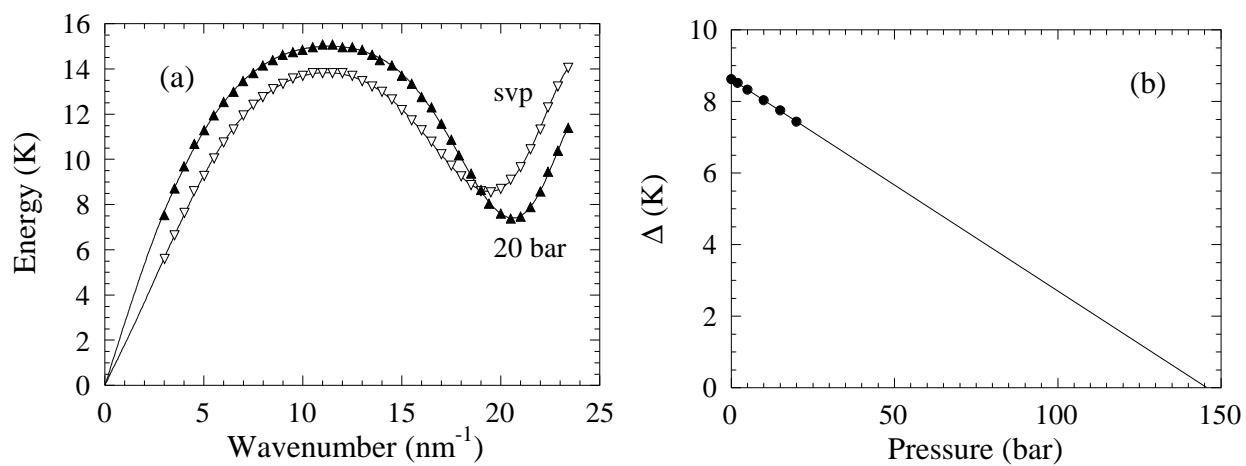

Figure 4. (a) Dispersion curve of elementary excitations in superfluid helium 4 at $0.5 \mathrm{~K}$, as obtained by neutron scattering [30]. Open triangles correspond to svp data and solid triangles to 20 bar; the solid lines are guides to the eye, and their slopes at the origin are deduced from the experimental sound velocity [31]. (b) Pressure variation of the roton gap $\Delta$; solid circles are experimental values from Ref. [30], and the solid line is a linear extrapolation at high pressure (see Sec. 4).

where $\lambda$ is a constant. As the spinodal is approached, the dispersion relation thus becomes quadratic, and we find near $P_{\mathrm{s}}$

$$
\alpha_{\mathrm{ph}}=\frac{35 \rho k_{\mathrm{B}}^{5 / 2} \zeta(5 / 2)}{64 \pi^{3 / 2} \hbar^{3 / 2}(2 \rho \lambda)^{3 / 4}} T^{3 / 2} \chi_{T}
$$

which diverges but remains negligible compared to $\alpha_{\mathrm{F}}$ at sufficiently low temperature. We also note that, if the sound remains adiabatic at small $k$ close to the spinodal, the use of the adiabatic (instead of isothermal) sound velocity, which does not vanish at $P_{\mathrm{S}}$, would further reduce the phonon contribution.

We have therefore shown that, in helium $3, \alpha$ is negative at low temperature near the spinodal, so that the spinodal pressure starts by decreasing with increasing temperature. In Sec. 2.1 we have reported experimental support for this behaviour; it also indicates that the spinodal pressure reaches a minimum value around $0.4 \mathrm{~K}$ and increases monotonically at higher temperature.

\section{Superfluid helium 4 at negative pressure}

\subsection{LANDAU MODEL AND THE DISPERSION CURVE}

Before discussing the case of negative pressure, we need to recall Landau's theory [28] which gives a qualitatively correct description of superfluid helium 4 at positive pressure. It is based on the dispersion relation of elementary excitations displayed in Fig. 4 (a); this shape was proposed 
by Landau [28] and measured by neutron scattering (see Refs. [29] and [30] for instance). There is a phonon branch at small momentum. A peculiarity of helium 4 is that it exhibits a structure at larger momentum, known as the maxon peak, and the roton minimum at $k_{0}$; the energy in the roton region can be approximated by:

$$
\varepsilon(k)=\Delta+\frac{\hbar^{2}\left(k-k_{0}\right)^{2}}{2 \mu}
$$

where $\Delta$ is the roton gap and $\mu$ the roton effective mass. Typical svp values for these parameters are: $\Delta=8.62 \mathrm{~K}, k_{0}=19.29 \mathrm{~nm}^{-1}$ and $\mu=0.161 m_{4}$ where $m_{4}=6.65 \times 10^{-27} \mathrm{~kg}$ is the mass of an atom of helium 4 [30]. The energy levels are populated by thermal activation, starting by the phonon branch at low temperature, and then filling the roton region at higher temperature. This model allows us to calculate the thermodynamic functions of the superfluid. It also gives an estimate of the superfluid transition temperature $T_{\lambda}$, when equating the total density to the density of normal fluid $\rho_{\mathrm{n}}$ coming from the excitations [29]:

$$
\rho_{\mathrm{n}}=-\frac{\hbar^{2}}{6 \pi^{2} k_{\mathrm{B}} T} \int_{0}^{+\infty} \frac{k^{4} \exp \left[\varepsilon(k) /\left(k_{\mathrm{B}} T\right)\right]}{\exp \left[\varepsilon(k) / k_{\mathrm{B}} T\right]-1} \mathrm{~d} k
$$

Unfortunately, this leads to an overestimate of $T_{\lambda}$ : for example, one would find $T_{\lambda}=2.76 \mathrm{~K}$ at svp, instead of the measured $2.17 \mathrm{~K}$. The discrepancy arises from the interaction between excitations; however, the model gives quantitatively correct results at low temperature (up to $1.6 \mathrm{~K}$ at svp) where there are few excitations. It also accounts for the experimentally observed decrease of $T_{\lambda}$ with increasing pressure. This comes from the decrease of the roton gap [32]. It has been measured by neutron scattering (see Ref. [30] for instance): the dispersion curves at low and high pressures are displayed on Fig. 4 (a), and $\Delta(P)$ in Fig. 4 (b). The shape of the dispersion curve has also been calculated using a density functional theory [33]. In the negative pressure region, Quantum Monte-Carlo calculations [34] show a trend of the maxon peak and the roton gap to merge together. This is also obtained in a recent extension of the density functional results to negative pressure [35].

We will therefore use Landau's simple model in the following, because it is valid at low temperatures, it allows analytical calculations, and captures the physics of superfluid helium 4.

\subsection{EXPANSION COEFFICIENT AND THE SPINODAL LINE}

The spinodal pressure of helium 4 at zero temperature has been estimated by several methods [3]; all of them give $P_{\mathrm{s}}$ around -10 bar. Density functional theory [11] and Quantum Monte-Carlo calculations [34] give a 

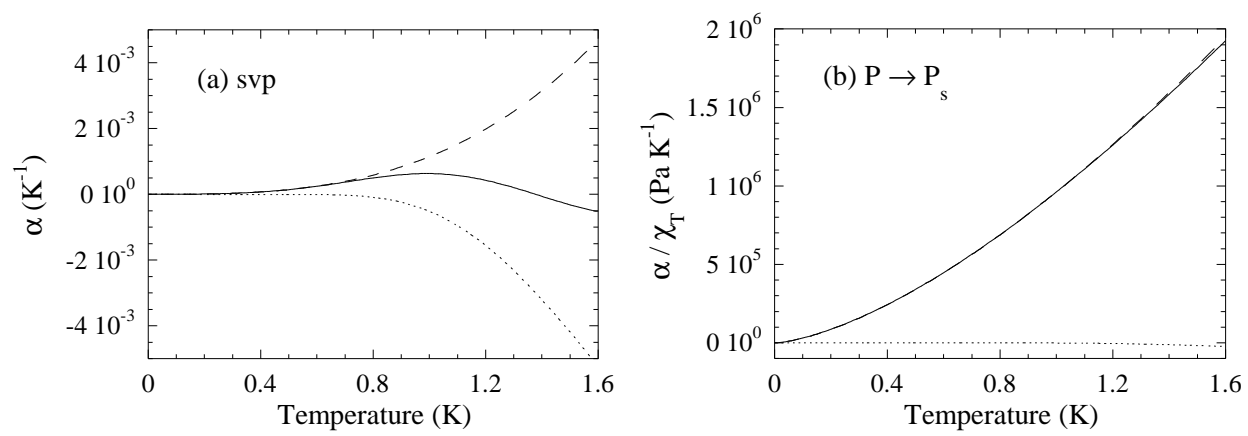

Figure 5. Expansion coefficient of superfluid helium 4 calculated with the Landau model (solid line) at saturated vapour pressure (a) and near $P_{\mathrm{s}}(\mathrm{b})$. The graphs also show the phonon contribution (dashed line) and the roton contribution (dotted line). In graph (b) each quantity is divided by $\chi_{\mathrm{T}}$ which diverges at $P_{\mathrm{s}}$.

monotonic spinodal line with positive slope. Hall and Maris [36] expect a change in slope of the spinodal line if a roton minimum still exists down to $P_{\mathrm{s}}$, and a monotonic spinodal if it disappears. In this section we will try to clarify this point by calculating the expansion coefficient near the spinodal.

Liquid helium 4 exhibits two lines of density extrema: a line of density minima (around $1 \mathrm{~K}$ at $\mathrm{svp}$ ) and a line of density maxima just above $T_{\lambda}$. The line of density minima was measured by Atkins and Edwards [37]; they found good agreement with Landau's model. Indeed, at positive pressure the phonon contribution to the expansion coefficient is given by Eq. 9, and the roton contribution is derived from Eqs. 4, 6, 8, and 12:

$$
\begin{aligned}
\alpha_{\text {rot }}= & -\frac{2 k_{\mathrm{B}}{ }^{1 / 2} \mu^{1 / 2} k_{0}^{2} \Delta}{(2 \pi)^{3 / 2} \hbar T^{1 / 2}}\left(1+\frac{3 k_{\mathrm{B}} T}{2 \Delta}\right) \exp \left(-\frac{\Delta}{k_{\mathrm{B}} T}\right) \\
& \times \chi_{T}\left\{\frac{\rho}{2 \mu}\left(\frac{\partial \mu}{\partial \rho}\right)_{T}+\frac{2 \rho}{k_{\mathrm{B}}}\left(\frac{\partial k_{0}}{\partial \rho}\right)_{T}-1\right. \\
& \left.\quad-\frac{\rho}{\Delta}\left(\frac{\partial \Delta}{\partial \rho}\right)_{T}\left[\frac{\Delta}{k_{\mathrm{B}} T}\left(1+\frac{\frac{3}{2}\left(\frac{k_{\mathrm{B}} T}{\Delta}\right)^{2}}{1+\frac{3 k_{\mathrm{B}} T}{2 \Delta}}\right)-1\right]\right\}
\end{aligned}
$$

The existence of a line of density minima is thus explained by the competition between these two terms (see Fig. 5 (a)): $\alpha_{\mathrm{ph}}$ is positive and is the leading term at low temperature; $\alpha_{\text {rot }}$ is negative and overcomes the phonon term at high temperature. The respective values at svp of the quantities $(\rho / \mu)(\partial \mu / \partial \rho)_{T},\left(\rho / k_{0}\right)\left(\partial k_{0} / \partial \rho\right)_{T}$, and $(\rho / \Delta)(\partial \Delta / \partial \rho)_{T}$ are -1.6, 0.41 , and -0.53 , as measured by neutron scattering [30]. These values were used to plot Fig. 5 (a); they differ slightly from the ones used by Atkins and Edwards [37]. 
We now extend this calculation to the negative pressure region. If the roton minimum still exists near the spinodal $\left(\right.$ at $\rho \simeq \rho_{\mathrm{s}}=94.82 \mathrm{~kg} \mathrm{~m}^{-3}$ ), Eq. 14 holds. We will use for the roton parameters in Eq. 14 the svp values, except for the gap for which we will take the approximate value $\Delta=10 \mathrm{~K}$. As for the phonons, we need to take into account the curvature of their dispersion relation as was pointed out in Sec. 2.2; this leads again to Eq. 11. We keep only the term involving $\Delta$ in the curly brackets of Eq. 14 and use the svp value for $(\partial \Delta / \partial \rho)_{T}$; this favours a larger negative $\alpha_{\text {rot }}$. The result near $P_{\mathrm{S}}$ is shown in Fig. $5(\mathrm{~b})$; we have plotted $\alpha / \chi_{T}$ to remove the divergence due to the spinodal. The total expansion coefficient is now positive up to temperatures where the Landau model ceases to be correct. We think this would also be the case if the roton minimum and the maxon peak were merging together.

We need to point out that all these calculations used a temperatureindependent value of $\rho_{\mathrm{s}}$; a self-consistent calculation, allowing a variation of $P_{\mathrm{s}}$ corresponding to the calculated $\alpha$ would be of interest, but we have not attempted it yet.

Our results support a monotonic spinodal in helium 4, the pressure of which increases with increasing temperature. They also show that the roton minimum does not need to disappear for the spinodal to be monotonic.

\subsection{SUPERFLUID TRANSITION}

Large attention has been given to the extension of the lambda line in the stretched liquid state. First, measurements of $T_{\lambda}$ have been performed at positive pressure below the saturated vapour pressure [38, 39]; they show a regular continuation of the lambda curve with the same negative slope. Skripov was the first to propose an extension at negative pressure [40]: he used a shifted Bose gas condensation line, so that the lambda line changes slope at negative pressure and $T_{\lambda}$ vanishes before the spinodal is reached. Campbell et al. [41] expected a multicritical point at $T=0 \mathrm{~K}$ between the spinodal and the lambda line because of phonon avalanche near the spinodal (due to the vanishing sound velocity). Hall and Maris [36] claimed that disappearance of the roton minimum would lead to a change in slope of the lambda line. Apenko proposed a Lindemann-like criterion for superfluidity of Bose liquids [42], leading to a transition line with a change in slope between high and low densities (liquid helium and Bose gas region respectively). Bauer et al. performed Quantum Monte-Carlo calculations [34]: they obtained a nearly constant $T_{\lambda}$ down to the spinodal. Finally, Skripov recently added to his picture the cases of the multicritical point and of a non-zero $T_{\lambda}$ at $P_{\mathrm{s}}[43]$.

We have used the Landau model to try to clarify this problem. Of course, as pointed out in Section 3.1, it does not give the exact $T_{\lambda}$; however, 

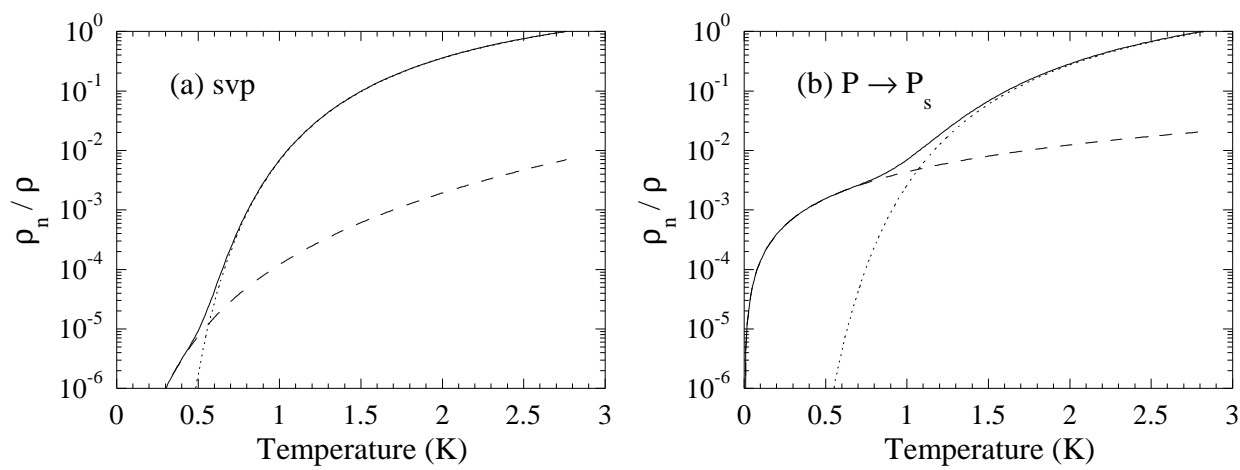

Figure 6. Normal fluid fraction in helium 4 calculated with the Landau model (solid line) at saturated vapour pressure (a) and near $P_{\mathrm{s}}(\mathrm{b})$. The graphs also show the phonon contribution (dashed line) and the roton contribution (dotted line).

this model is valid at low temperature, and with the input of the roton parameters, it predicts correctly the slope of the lambda line, so that it will provide some understanding of what is happening at negative pressure. Using Eq. 13, one can calculate the phonon and roton contributions $\left(\rho_{\mathrm{n}-\mathrm{ph}}\right.$ and $\rho_{\mathrm{n}-\text { rot }}$ respectively) to the normal fraction $\rho_{\mathrm{n}} / \rho$. The result at svp is shown in Fig. 6 (a): $\rho_{\mathrm{n}-\mathrm{ph}}$ dominates at low temperature, and it is overcome by $\rho_{\mathrm{n} \text {-rot }}$ around $0.6 \mathrm{~K}$; the fluid becomes normal at $T_{\lambda}=2.76 \mathrm{~K}$. If one used a linear phonon branch, the vanishing sound velocity at the spinodal would lead to a phonon avalanche and a multicritical point as proposed by Campbell et al. [41]; however, the curvature of the dispersion relation (Eq. 10) prevents this phenomenon to occur and our result is shown in Fig. 6 (b). One can see that $\rho_{\mathrm{n}-\mathrm{ph}}$ is much higher than at svp but remains less than $2 \%$ up to $T_{\lambda}$. For $\Delta=10 \mathrm{~K}$ and $\rho=\rho_{\mathrm{s}}=94.82 \mathrm{~kg} \mathrm{~m}^{-3}$, we obtain $T_{\lambda}=2.81 \mathrm{~K}$. Concerning the slope of the lambda line, we find here that it remains negative, but choosing $\Delta=9.5 \mathrm{~K}$ instead of $10 \mathrm{~K}$ would lead to $T_{\lambda}=2.65 \mathrm{~K}$ near $P_{\mathrm{s}}$, and thus to a change in slope. This comes from a competition when one approaches $P_{\mathrm{s}}$ between the increase of $\Delta$ which tends to increase $T_{\lambda}$, and a decrease of $\rho$ and increase in $\rho_{\mathrm{n}-\mathrm{ph}}$ which tend to lower $T_{\lambda}$.

Our results show that $T_{\lambda}$ remains finite down to the spinodal; we would like to emphasize that this comes from the curvature of the phonon branch, and that it is obtained at low temperature where Landau's theory is valid with no approximation. Our calculations are in qualitative agreement with Quantum Monte-Carlo simulations [34]. They also indicate that the lambda line may change slope even if a slight roton minimum remains near $P_{\mathrm{s}}$. 


\section{Overpressurized superfluid helium 4}

Finally, we turn to the other metastable region for the liquid, at pressures above the melting pressure $P_{\mathrm{m}}$. Because the liquid-solid transition is first order as the liquid-gas transition, it is possible to observe a metastable overpressurized liquid. Previous experiments only reached an overpressure of a few millibars, but recently we were able to achieve an overpressure of 4.3 bar with a modified version of our cavitation experiment [3, 44]. A theoretical description of the thermodynamics in this region is thus needed.

\subsection{INSTABILITY OF THE SUPERFLUID AGAINST THE SOLID}

We reconsider a theory proposed by Schneider and Enz [45]. They have suggested that the limit of metastability of the superfluid against the solid is associated with the softening of the roton mode. The roton minimum, which is known to decrease with increasing pressure (see Section 3.1, Fig. 4 (b)), would vanish at some pressure $P_{\Delta=0}>P_{\mathrm{m}}$; the liquid would then become unstable against a density fluctuation of finite wave number $k_{0}$, which is close to the reciprocal-lattice vector of solid helium. A linear extrapolation of neutron scattering data for the roton gap (Fig. 4 (b)) leads to an estimate of 150 bar for the stability limit pressure. Edwards and Maris have recently obtained $P_{\Delta=0} \simeq 200$ bar within a density functional approach [35].

\subsection{SUPERFLUID TRANSITION}

As in the case of the stretched liquid, we may consider the continuation of the lambda line in the overpressurized region. This was mentioned by Skripov [40], but no attempt was made to determine the behaviour of this metastable lambda line.

To do this, we use the Landau model up to the limit where the roton gap vanishes. As the pressure increases above $P_{\mathrm{m}}$, the roton gap decreases and the lambda line should continue with a negative slope as below $P_{\mathrm{m}}$. An interesting issue is to know whether $T_{\lambda}$ reaches 0 before the roton instability occurs. Of course, we need to use the simple Landau model with caution, because its range of validity is reduced to lower temperature as $P_{\Delta=0}$ is approached; indeed, to neglect interactions requires a small number of elementary excitations. For any $P<P_{\Delta=0}$, this condition will be fulfilled at sufficiently low temperature; we can use without any approximation the usual formulas for $\rho_{\mathrm{n}-\mathrm{ph}}$ and $\rho_{\mathrm{n} \text {-rot }}$. These show that close enough to the absolute zero, phonons will dominate the thermodynamics: $\rho_{\mathrm{n}} \simeq \rho_{\mathrm{n}-\mathrm{ph}} \ll \rho$; as the sound velocity increases with pressure, $\rho_{\mathrm{n}-\mathrm{ph}}$ will even decrease. Therefore metastable liquid helium close to the line $T=0$ remains superfluid up to $P_{\Delta=0}$; this is a first important result. 
We now use the Landau model to predict $T_{\lambda}$, as we did in Section 3.3, keeping in mind that this will not give quantitatively correct results, but will provide a qualitative picture. The main contribution to the normal density near $T_{\lambda}$ is due to rotons. Assuming that the energy in the roton region remains quadratic (Eq. 12) [46] and that the effective mass does not go to zero, this gives a vanishing $T_{\lambda}$ at $P_{\Delta=0}$. We think that this result will not be qualitatively affected by interactions, because the Landau model gives an overestimate of $T_{\lambda}$. We conclude that the lambda line meets the $P_{\Delta=0}$ line at $T=0 \mathrm{~K}$.

\section{Conclusion}

We have shown that in addition to its purity, the quantum nature of helium makes it an ideal system in which to study metastability. Indeed, quantum statistics give a powerful theoretical tool to investigate the metastable regions. In the case of helium 3, they allowed us to explain the origin of the minimum observed in the spinodal line. We are presently adapting our experimental setup to check the existence of such a minimum in liquid water [47]. The use of an analytically tractable model to describe superfluid helium 4 allowed us to predict a monotonous spinodal in this other case. It also gives a qualitative picture for the extension of the lambda line in the metastable regions; further work is in progress to take into account interactions between elementary excitations and make the picture quantitative $[35,48]$. In view of our recent experiments on overpressurized liquid helium 4 , we have also considered an instability related to the softening of the roton mode.

\section{Acknowledgements}

We would like to thank H.J. Maris for stimulating discussions.

\section{References}

1. Lambaré, H., Roche, P., Balibar, S., Maris, H.J., Andreeva, O.A., Guthmann, C., Keshishev, K.O., and Rolley, E. (1998) Cavitation in superfluid helium-4 at low temperature, Eur. Phys. J. 2, 381-391.

2. Caupin, F. and Balibar, S. (2001) Cavitation pressure in liquid helium, Phys. Rev. B 64, 064507 (1-10).

3. Balibar, S. and Caupin, F. (2002) The limits of stability of liquid helium, this conference, and references therein.

4. Maris, H.J. (1995) Theory of quantum nucleation of bubbles in liquid helium, J. Low Temp. Phys. 98, 403-424. 
5. Guilleumas, M., Barranco, M., Jezek, D.M., Lombard, R.J., and Pi, M. (1996) Quantum cavitation in liquid helium, Phys. Rev. B 54, 16135-16138.

6. Caupin, F. and Balibar, S. (2000) Search for quantum cavitation in liquid helium 3, Physica B 284-288, 212-213.

7. Caupin, F., Balibar, S., and Maris, H.J. (2002) Nucleation in a Fermi liquid at negative pressure, J. Low Temp. Phys. 126, 91-96.

8. Speedy R.J. (1982) Stability-limit conjecture. An interpretation of the properties of water. J. Phys. Chem. 86, 982-991.

9. Caupin, F., Balibar, S., and Maris, H.J. (2001) Anomaly in the stability limit of liquid helium 3, Phys. Rev. Lett. 87, 145302 (1-4).

10. Xiong, Q. and Maris, H.J. (1989) Liquid helium at negative pressure : nucleation of bubbles and anomalous phonon dispersion, J. Low Temp. Phys. 77, 347-369.

11. Guilleumas, M., Pi, M., Barranco, M., Navarro, J., and Solís, M.A. (1993) Thermal nucleation of cavities in liquid helium at negative pressures, Phys. Rev. B 47, 91169119.

12. Pettersen, M.S., Balibar, S., and Maris, H.J. (1994) Experimental investigation of cavitation in superfluid ${ }^{4} \mathrm{He}$, Phys. Rev. B 49, 12062-12070.

13. The expression of $V \tau$ obtained in Ref. [12] actually depends on the minimum pressure reached, but we have checked that this does not affect our conclusions.

14. Boghosian, C., Meyer, H., and Rives, J.E. (1966) Density, coefficient of thermal expansion, and entropy of compression of liquid helium-3 under pressure below 1.2 K, Phys. Rev. 146, 110-119.

15. Maris, H.J. (1994) Nucleation of bubbles on quantized vortices in helium-4, J. Low Temp. Phys. 94, 125-144.

16. Abraham, B.M., Chung, D., Eckstein, Y., Ketterson, J.B., and Roach, P.R. (1972) Sound propagation, density and viscosity in liquid ${ }^{3} \mathrm{He}$, J. Low Temp. Phys. 6, 521-528.

17. Roach P.R., Eckstein, Y., Meisel, M.W., and Aniola-Jedrzejek, L. (1983) Thermal expansion, velocity of sound, and compressibility in liquid ${ }^{3} \mathrm{He}$ under pressure, J. Low Temp. Phys. 52, 433-447.

18. Thermodynamically, the spinodal line is derived from the isothermal sound velocity rather than from the adiabatic one. Both velocities lead to spinodal lines showing a minimum and their difference is less than 30 mbar. We have actually plotted the spinodal obtained with the isothermal data in Fig. 2, and we used it in our discussion.

19. Debenedetti, P.G. (1996) Metastable liquids, Princeton University Press, Princeton, and references therein.

20. Ahlers, G. (1976) Experiments near the superfluid transition in ${ }^{4} \mathrm{He}$ and ${ }^{3} \mathrm{He}-{ }^{4} \mathrm{He}$ mixtures, in K.H. Bennemann and J.B. Ketterson (eds.), The physics of liquid and solid helium, John Wiley and Sons, New York, Part I, Chap. 2, 85-206.

21. Lee, D.M., Reppy, J.D., and Fairbank, H.A. (1958) Evidence for a density maximum in liquid $\mathrm{He}^{3}$ near 0.5 K, Bull. Am. Phys. Soc. Ser. II. 3, 339.

22. Brueckner, K.A. and Atkins, K.R. (1958) Coefficient of thermal expansion of liquid $\mathrm{He}^{3}$ near 0.5 K, Phys. Rev. Lett. 1, 315-318.

23. Landau, L.D. (1956) The theory of a Fermi liquid, Zh. Eksper. Teor. Fiz., 30, 1058-1064 [(1957) Sov. Phys. JETP 3, 920-925].

24. Greywall, D.S. (1986) ${ }^{3}$ He specific heat and thermometry at millikelvin temperatures, Phys. Rev. B 33, 7520-7538.

25. Balibar, S., Caupin, F., Roche, P., and Maris, H.J. (1998) Quantum cavitation: a 
QUANTUM STATISTICS OF METASTABLE LIQUID HELIUM 15

comparison between superfluid helium-4 and normal liquid helium-3, J. Low Temp. Phys. 113, 459-471.

26. Greywall, D.S. (1983) Specific heat of normal liquid ${ }^{3}$ He, Phys. Rev. B 27, 27472766.

27. Lifshitz, I.M. and Kagan, Y. (1972) Quantum kinetics of phase transitions at temperatures close to absolute zero, Zh. Eksp. Teor. Fiz. 62, 385-402 [(1972) Sov. Phys. JETP 35, 206-214]. See also Ref. [10].

28. Landau, L.D. (1947) On the theory of superfluidity of helium II, J. Phys. (Mosc.) 11, 91.

29. Wilks, J. (1967) The properties of liquid and solid helium, Clarendon Press, Oxford.

30. Gibbs, M.R., Andersen, K.H., Stirling, W.G., and Schober, H. (1999) The collective excitations of normal and superfluid ${ }^{4} \mathrm{He}$ : the dependence on pressure and temperature, J. Phys.: Condens. Matter 11, 603-628.

31. Abraham, B.M., Eckstein, Y., Ketterson, J.B., Kuchnir, M., and Roach, P.R. (1970) Velocity of sound, density and Grüneisen constant in liquid ${ }^{4} \mathrm{He}$, Phys. Rev. A 1, 250-257.

32. Atkins, K.R. (1955) Slope of the $\lambda$ curve of liquid helium, Phys. Rev. 98, 319-320.

33. Dalfovo, F., Lastri, A., Pricaupenko, L., Stringari, S., and Treiner, J. (1995) Structural and dynamical properties of superfluid helium: a density-functional approach, Phys. Rev. B 52, 1193-1209.

34. Bauer, G.H., Ceperley, D.M., and Goldenfeld, N. (2000) Path-integral Monte Carlo simulation of helium at negative pressures, Phys. Rev. B 61, 9055-9060.

35. Edwards, D.O. and Maris, H.J. (unpublished).

36. Hall, S.C. and Maris, H.J. (1997) Thermodynamics and nucleation of bubbles in normal and superfluid liquid helium-4 at negative pressures, J. Low Temp. Phys. 107, 263-282.

37. Atkins, K.R. and Edwards, M.H. (1955) Coefficient of expansion of liquid helium II, Phys. Rev. 97, 1429-1434.

38. Rybarcyk, L.J. and Tough, J.T. (1981) Superheating in He II and the extension of the lambda line, J. Low Temp. Phys. 43, 197-202.

39. Nishigaki, K. and Saji, Y. (1986) Superheating in He II and successive phase transitions in metastable states, Phys. Rev. B 33, 1657-1662.

40. Skripov, V.P. (1994) The metastability boundary in the ${ }^{4}$ He diagram of state, $Z h$. Fiz. Kh. 68, 1382-1385 [(1994) Russ. J. Phys. Chem 68, 1252-1255].

41. Campbell, C.E., Folk, R., and Krotscheck, E. (1996) Critical behavior of liquid ${ }^{4} \mathrm{He}$ at negative pressures, J. Low Temp. Phys. 105, 13-36.

42. Apenko, S.M. (1999) Critical temperature of the superfluid transition in Bose liquids, Phys. Rev. B 60, 3052-3055.

43. Skripov, V.P. (2000) Extension of the $\lambda$ curve of ${ }^{4} \mathrm{He}$ into the region of the metastable state of liquid helium, Usp. Fiz. Nauk 170, 559-563 [Phys. Usp. 43, 515-519].

44. Chavanne, X., Balibar, S., and Caupin, F. (2001) Acoustic nucleation of solid helium 4 on a clean glass plate, J. Low Temp. Phys. 125, 155-164.

45. Schneider, T. and Enz, C.P. (1971) Theory of the superfluid-solid transition of ${ }^{4} \mathrm{He}$, Phys. Rev. Lett. 27, 1186-1188.

46. Notice that, unlike the case of phonons, the curvature of the dispersion curve is accounted for in the usual calculation of $\rho_{\mathrm{n}-\mathrm{rot}}$.

47. Caupin, F. and Fourmond, V. (2002) Ultrasonic cavitation in freon at room temperature, this conference.

48. Caupin, F. and Maris, H.J. (unpublished). 
